\title{
Gender Differences in the Association Between Cyberbullying Victimization and Perpetration: The Role of Anger Rumination and Traditional Bullying Experiences
}

\author{
Ágnes Zsila ${ }^{1,2}$ • Róbert Urbán ${ }^{2}$ • Mark D. Griffiths ${ }^{3,4}$. \\ Zsolt Demetrovics ${ }^{2}$
}

\begin{abstract}
Studies investigating the similarities and differences in traditional bullying and cyberbullying experiences have demonstrated considerable gender differences concerning its determinants. The aim of the present study was to provide further evidence for the differential role of determinants for males and females by investigating the moderating role of traditional bullying and anger rumination in the relationship of past cyberbullying victimization and recent cyberbullying perpetration in respect to gender. A total of 1500 Hungarian adolescents and adults $\left(57.9 \%\right.$ male, $M_{\text {age }}=28.9$ years, $\left.S D=8.7\right)$ completed an online survey on bullying experiences. Results indicated that males were more likely than females to engage in cyberbullying when they had been previously bullied online. Furthermore, high anger rumination elevated the risk of perpetration among male cyberbullying victims, while repeated victimization in traditional bullying increased the risk of cyberbullying perpetration among females. These results underline the importance of considering gender differences in intervention efforts against bullying.
\end{abstract}

Keywords Anger rumination · Cyberbullying · Online bullying · Perpetration · Traditional bullying · Cyberharassment

\section{Mark D. Griffiths}

mark.griffiths@ntu.ac.uk

1 School of Psychology, ELTE Eötvös Loránd University, Budapest, Hungary

2 Institute of Psychology, ELTE Eötvös Loránd University, Budapest, Hungary

3 Psychology Department, Nottingham Trent University, Nottingham, UK

4 International Gaming Research Unit, Psychology Department, Nottingham Trent University, 50 Shakespeare Street, Nottingham NG1 4FQ, UK 
Bullying has been defined as a repeated aggressive act that occurs when one or more individuals harass, mistreat, or exclude someone in order to inflict harm (Olweus 1999). To date, it has been broadly studied for its negative effects on the physical and mental health of young individuals (Aluede et al. 2008; Ortega et al. 2009). Technological advancements have led to the emergence of new forms of bullying and harassment (e.g., cyberbullying, online trolling, sending harassing messages via social media, sharing embarrassing photos and videos online without permission) (Griffiths 2014). Many of these newer forms are characterized by anonymity and in most cases as these aggressive and harassing acts are performed using electronic devices via social media and online games (Thacker and Griffiths 2012; Tokunaga 2010). Although it has been suggested that the prevalence of cyberbullying (CB) might exceed that of traditional bullying (TB), recent studies have yet to confirm such speculation (e.g., Kowalski et al. 2014; Olweus 2012; Ortega et al. 2009).

According to a recent meta-analysis by Modecki et al. (2014), 36\% of adolescents have been victimized in CB (ranging from 2.2 to $56.2 \%$ ) and 15\% in TB (ranging from 9 to $97.9 \%$ ). Very few studies have investigated the prevalence of CB among adults (e.g., Balakrishnan 2015; MacDonald and Roberts-Pittman 2010). Balakrishnan (2015) reported that $39.7 \%$ of adults had been bullied and $33.6 \%$ bullied others online, while MacDonald and RobertsPittman (2010) reported that $21.9 \%$ of adults had experienced CB as a victim and $8.6 \%$ as a perpetrator. Previous studies have found strong associations between TB and CB victimization and perpetration (e.g., Li 2007; Smith et al. 2008), suggesting that experiences in one form can possibly predict the involvement in another form (Dooley et al. 2009; Smith et al. 2008). The present study investigates the role of repeated TB experiences and the association between past $\mathrm{CB}$ victimization and recent $\mathrm{CB}$ perpetration with respect to gender differences. Anger has been identified as an important psychological determinant in this association (Camodeca et al. 2002; Dooley et al. 2009; Hemphill et al. 2012); therefore, anger rumination was also considered in the present investigation as a possible determinant of perpetration.

In relation to gender differences in the prevalence rates of $\mathrm{TB}$ and $\mathrm{CB}$, most studies have found that males are more likely to be victimized in TB than females (e.g., Huang and Chou 2010; Wang et al. 2009), while females are more likely to be victimized in CB than males (e.g., DeHue et al. 2008; Kowalski and Limber 2007; Ortega et al. 2009). However, the proportion of males was higher among both TB and CB perpetration (e.g., Chan and Wong 2016; Lee and Shin 2017). Furthermore, males have been found to bully others more often and repetitively than females (Buelga et al. 2015; Kowalski et al. 2010).

Most studies investigating gender differences have only focused on prevalence rates, without examining the psychological determinants of bullying that might also be affected by gender (Wong et al. 2017). Erdur-Baker (2010) noted that there might be differences in the association between TB and CB experiences across gender. For instance, it has been found that females perceive TB (Hughes et al. 2009) and CB (Campbell et al. 2013) to be more hurtful than males. Furthermore, males are more likely than females to exhibit online aggression if they have experienced CB as a victim (Wong et al. 2014; Wong et al. 2017), while females are more likely to engage in $\mathrm{CB}$ as a reaction to their victimization in traditional forms of aggression (Yang et al. 2013). These findings suggest that the motives of perpetrators may also differ across gender (Wong et al. 2017). In line with this, Barlett and Coyne (2014) suggested that gender should be treated as a moderator when investigating the psychological determinants and motives of CB perpetration and victimization.

In other research, Kowalski and Limber (2013) emphasized that TB and CB should not be approached as two separate phenomena given that there is a considerable overlap between TB 
and CB experiences (Fletcher et al. 2014; Hemphill and Heerde 2014; Waasdorp and Bradshaw 2015). For instance, it has been proposed that TB victimization might motivate some individuals to engage in $\mathrm{CB}$ as a form of revenge within the safe confines of anonymity (Dooley et al. 2009; Hemphill et al. 2012). Previous experiences of aggression in TB might also lead to the exacerbation of aggressive behaviors in cyberspace (Li 2007; Smith et al. 2008). Studies investigating gender differences in delinquency suggest that the underlying mechanisms of participation in violent behaviors are the same for males and females (LaGrange and Silverman 1999). Furthermore, it has been found that male and female delinquency share the same risk factors (e.g., antisocial attitudes, educational problems, poor family attachment), providing further confirmation that the psychological mechanisms of offending might not differ across gender (Simourd and Andrews 1994). Drawing on these findings, in the present study, it was expected that previous $\mathrm{CB}$ victims with repetitive experiences of TB as a bully (both past and recent) would be more likely to bully others in cyberspace irrespective of gender. However, taking into account that females appear to be more distressed by bullying experiences than males (Campbell et al. 2013; Fenaughty and Harré 2013) and that females are more likely to engage in CB as a reaction to traditional forms of aggression (Yang et al. 2013), it was expected that past CB victims with repetitive experiences of TB as a victim (i.e., who were bullied in the past) would have an increased risk of recent $\mathrm{CB}$ offending, and that the role of repeated TB victimization in this association would only be found among females.

With regard to anger, males have been found to have a higher tendency of exhibiting direct forms of aggression and confrontation when facing a stressful event, whereas females are more likely to respond to stress with a "tend and befriend" reaction, which urges avoidance (Taylor et al. 2000; Turton and Campbell 2005). According to previous studies, the most common response to CB is anger (Camodeca et al. 2002; Ortega et al. 2012). Anger rumination which is defined as a tendency to recall and ruminate about past experiences of anger (Sukhodolsky et al. 2001) has been directly associated with aggression (Bushman et al. 2005; Maxwell 2004). Some studies have failed to find any gender differences in anger rumination (Maxwell 2004; Sukhodolsky et al. 2001), while other studies have reported males as having a higher level of anger rumination (Maxwell et al. 2005; Peled and Moretti 2010). Based on these associations, it was expected that past $\mathrm{CB}$ victims with a tendency to ruminate on past anger experiences would be more likely to bully others online than victims without high anger ruminative tendencies, and that the role of anger rumination in this association would only be found among males.

\section{Aims of the Present Study}

Previous studies have reported an association between TB and CB experiences (e.g., Hinduja and Patchin 2008; Kowalski et al. 2012), although research suggests that there are considerable gender differences in the perception, motivation, and reaction related to bullying experiences (Barlett and Coyne 2014; Wong et al. 2017). There is a lack of research investigating the role of $\mathrm{TB}$ experiences and anger in the association between $\mathrm{CB}$ victimization and perpetration in respect to gender differences. Therefore, the present study investigated the moderating role of repeated $\mathrm{TB}$ experiences and anger rumination in the association between past $\mathrm{CB}$ victimization (i.e., during victim's lifetime, excluding the past year) and recent CB perpetration (i.e., within the past year) across gender in a sample of Hungarian adolescents and adults. The 
exploration of gender differences in these associations will advance the understanding of individual factors that might elevate the risk of engaging in online aggression. Based upon the previous literature, the following hypotheses were proposed: (i) gender will moderate the relationship between past $\mathrm{CB}$ victimization and recent $\mathrm{CB}$ perpetration; more specifically, male $\mathrm{CB}$ victims will be more likely to bully others online than females; (ii) anger rumination will moderate the relationship between past $\mathrm{CB}$ victimization and recent $\mathrm{CB}$ perpetration among males; (iii) repeated TB perpetration will moderate this association irrespective of gender; and (iv) repeated TB victimization will moderate this association among females.

\section{Methods}

\section{Participants and Procedure}

A total of 1500 adolescents and adults $\left(57.9 \%\right.$ male, $M_{\text {age }}=28.9$ years, $\left.S D=8.7\right)$ participated in the study. Data collection was conducted via an online questionnaire during the spring of 2017. The call for participation was advertised on two popular Hungarian websites focusing on news and gaming (444.hu and GameStar.hu). Participants were informed about the purpose of the investigation (i.e., exploring bullying experiences) and that participation is voluntary. For underage participants (those below age 18 years), parental approval was required. As an incentive, six 20,000 HUF-worth gift vouchers (approximately \$77 US) were raffled among those participants who completed the questionnaire. Ethical approval was gained from the Institutional Review Board of the research team's university and the present investigation was conducted in accordance with the Declaration of Helsinki.

\section{Measures}

Data on the key sociodemographic characteristics were collected (i.e., gender and age).

Traditional Bullying and Cyberbullying Experiences First, participants were introduced to the definition of traditional bullying (TB) (Shaw et al. 2013) and cyberbullying (CB) (Tokunaga 2010). Second, they were asked whether they experienced TB and/or CB in the past year and/or in their lifetime (excluding past year). If participants responded positively to either of these questions, they were asked to indicate their roles in these experiences (i.e., victim, perpetrator, or both) and the frequency of their experiences in the past year $(1=$ less than once a month, $2=$ once or twice a month, $3=$ weekly-biweekly, $4=$ several times a week). This latter variable was linearized in the data analysis (times per month) for both TB and $\mathrm{CB}$ experiences. Finally, participants were asked to indicate which of the two actions (i.e., $\mathrm{TB}$ or $\mathrm{CB}$ ) they considered more harmful. They rated both actions on a 10-point Likert scale.

Anger Rumination Participants' tendency to recall and think about past experiences of anger was assessed with the Anger Rumination Scale (ARS; Sukhodolsky et al. 2001). Two study-relevant subscales were used: angry afterthoughts (six items; e.g., "Memories for even minor annoyances bother me for a while") ( $\alpha=0.87$ in the present study) and angry memories (five items; e.g., "I ruminate about my past anger experiences") $(\alpha=0.85)$. Participants were asked to indicate the extent to which the statements were characteristic of them on a 4-point Likert scale anchored by $1=$ "almost never" and $4=$ "almost always." The translation of items 
into Hungarian was carried out in accordance with the protocol recommended by Beaton et al. (2000). A strong correlation was observed between the two ARS subscales $(r=0.83$; $p<0.001)$; therefore, an average score of them was calculated and used in the data analysis $(\alpha=0.91)$.

\section{Statistical Analysis}

Data analysis was conducted using SPSS version 22.0 (IBM SPSS Inc., Chicago, Illinois) with PROCESS macro version 2.16.3 (Hayes 2013). For group comparisons, chi-square tests, $t$ tests, and one-way ANOVAs were calculated. Effect sizes (Hedges' $g$ and Phi) are being interpreted in the following way: $0.2=$ small effect, $0.5=$ medium effect, $0.8=$ large effect for Hedges' $g$ (Cohen 1988) and $0.1=$ small effect, $0.3=$ medium effect, $0.5=$ large effect for Phi (Field 2009). In the moderation models, CB victimization during participants' lifetime (dichotomous variable; 0 = not victimized, $1=$ victimized) was the independent variable (i.e., past $\mathrm{CB}$ victim), whereas $\mathrm{CB}$ perpetration in the past year (dichotomous variable; $0=$ not being a perpetrator, $1=$ being a perpetrator) was the outcome variable (i.e., recent $\mathrm{CB}$ perpetrator). Age was entered as a covariate in the moderation analyses. To test the possibly differential effect of anger rumination (continuous variable) and past TB experiences as a victim (multicategorical variable; $0=$ not victimized, $1=$ victimized either in the past year or during their lifetime [i.e., non-recidivist victim], 2 = victimized both in the past year and during their lifetime [i.e., recidivist victim]) or as a perpetrator (multicategorical variable; $0=$ not being a perpetrator, $1=$ being a perpetrator either in the past year or during their lifetime [i.e., non-recidivist perpetrator], $2=$ being a perpetrator both in the past year and during their lifetime [i.e., recidivist perpetrator]) across gender, moderation models were conducted for male and female participants separately.

\section{Results}

\section{Descriptive Statistics}

Almost half of participants (47.2\% of the total sample) experienced TB during their lifetime (excluding past year), whereas approximately one quarter of participants $(28.0 \%)$ reported of any $\mathrm{CB}$ experiences during their lifetime. Therefore, past TB experiences were more frequently reported by participants than past CB experiences $\left(\chi^{2}(1)=117.84 ; p<0.001 ; P h i=0.20\right)$. In contrast, one third of participants (33.3\%) reported of CB experiences in the past year, whereas less than one quarter of participants (23.93\%) reported of TB experiences in the past year, indicating that recent $\mathrm{CB}$ experiences have been more prevalent among participants than recent TB experiences $\left(\chi^{2}(1)=32.43 ; p<0.001 ; P h i=0.10\right)$. However, effect sizes were small.

\section{Group Comparisons Across Gender}

Significant gender differences were observed within different TB $\left(\chi^{2}(1)=42.37 ; p<0.001\right.$; $P h i=0.25$ for past experiences and $\chi^{2}(1)=47.48 ; p<0.001 ; P h i=0.36$ for recent experiences) and CB roles $\left(\chi^{2}(1)=96.37 ; p<0.001 ; P h i=0.48\right.$ for past experiences and $\chi^{2}(1)=73.55$; $p<0.001$; Phi $=0.38$ for recent experiences) (see Table 1). According to the post hoc $z$ tests of chi-square tests, victimization was more frequently reported among females than among males 


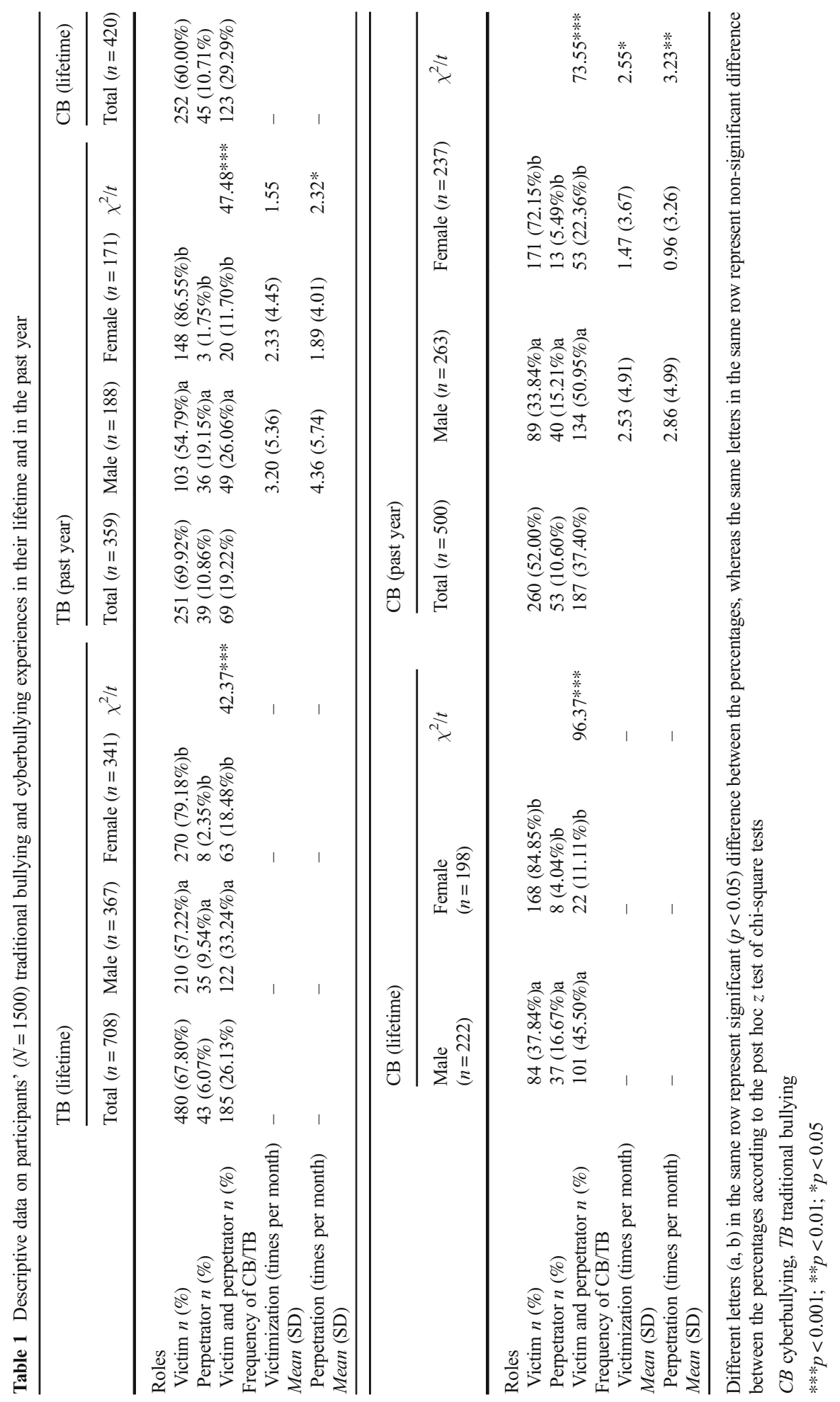


in both $\mathrm{TB}$ and $\mathrm{CB}$. In contrast, the proportion of male perpetrators was higher than the proportion of female perpetrators in both TB and CB. Similarly, males were more likely to experience $\mathrm{TB}$ and $\mathrm{CB}$ in both roles (i.e., victim and perpetrator) than females.

Male TB perpetrators bullied others more frequently during the past year than female TB perpetrators $(t(46)=2.32 ; p=0.03 ;$ Hedges' $g=0.50)$, and the same association was observed in relation to $\mathrm{CB}$ perpetration with a medium effect size $(t(132)=3.23 ; p=0.002$; Hedges' $g=$ $0.41)$. Likewise, male $\mathrm{CB}$ victims were more frequently bullied online than female victims in the past year $(t(403)=2.55 ; p=0.01 ;$ Hedges' $g=0.21)$. However, the effect size was small.

Significant gender differences were found in the perceived harmfulness of TB and CB and in anger rumination (see Table 2). Overall, participants perceived TB more harmful than $\mathrm{CB}$ $(t(1488)=13.35 ; p<0.001 ;$ Hedges' $g=0.38)$. The effect size was small to medium. Females perceived both TB and CB more harmful than males, and the effect size was medium to large (Hedges' $g$ was 0.44 for TB and 0.70 for CB). Furthermore, females yielded higher scores on the ARS anger rumination scale than males. However, the effect sizes were generally weak for the subscales and the total score (Hedges' $g$ was between 0.21 and 0.28).

When comparing $\mathrm{TB}$ and $\mathrm{CB}$ victims and perpetrators, non-overlapping categories were used in the analysis in order to examine clear associations between roles, perceived harmfulness of actions, and ruminative tendencies. It was found that TB victims perceived TB to be more harmful than TB perpetrators (Welch $(56)=4.20 ; p=0.009)$, and the effect size was large (Hedges' $g=0.86$ ). Marginally significant difference was found between TB victims and CB perpetrators in their perception of CB's harmfulness $($ Welch $(56)=2.79 ; p=0.05)$, indicating that $\mathrm{TB}$ victims found $\mathrm{CB}$ to be more harmful than $\mathrm{CB}$ perpetrators (Hedges' $g=0.57$ ). The effect size was again medium to large. In contrast, no significant difference was observed between $\mathrm{TB}$ and $\mathrm{CB}$ victims and perpetrators in anger rumination.

\section{Group Comparisons Across Recidivist and Non-recidivist Victims and Perpetrators of Traditional Bullying and Cyberbullying}

Significant differences were observed when investigating the associations of sociodemographic characteristics, participants' perception of the harmfulness of TB and CB, anger rumination, and repeated and non-repeated victimization and perpetration (see Table 3).

In relation to $\mathrm{TB}$ experiences, recidivist TB victims (i.e., those who were victimized both in their lifetime and in the past year) yielded higher scores on the angry memories subscale of the ARS $(t(490)=2.57 ; p=0.01 ;$ Hedges' $g=0.21)$ and on the two averaged subscales of the ARS $(t(496)=2.13 ; p=0.03 ;$ Hedges' $g=0.17)$ compared to non-recidivist TB victims (i.e., those who were victimized either in their lifetime or in the past year). However, the effect sizes were small. It was also found that recidivist TB perpetrators perceived both TB $(t(109)=3.85$; $p<0.001$; Hedges' $g=0.61)$ and CB $(t(125)=5.01 ; p<0.001$; Hedges' $g=0.73)$ less harmful than non-recidivist TB perpetrators. The effect sizes were large.

With regard to $\mathrm{CB}$ experiences, the proportion of males was higher among recidivist $\mathrm{CB}$ victims than among non-recidivist $\mathrm{CB}$ victims $\left(\chi^{2}(1)=7.34 ; p=0.007 ; P h i=0.12\right)$, and recidivist $\mathrm{CB}$ victims were relatively older than non-recidivist $\mathrm{CB}$ victims $(t(527)=1.94$; $p=0.05$; Hedges' $g=0.17$ ). However, the effect sizes were small. The results also indicated that the proportion of males was higher among recidivist $\mathrm{CB}$ perpetrators than among nonrecidivist $\mathrm{CB}$ perpetrators $\left(\chi^{2}(1)=7.98 ; p=0.006 ; P h i=0.19\right)$. Furthermore, recidivist $\mathrm{CB}$ perpetrators perceived CB less harmful than non-recidivist $\mathrm{CB}$ perpetrators $(t(223)=3.30 ; p=$ 0.001 ; Hedges' $g=0.44$ ). The effect size was medium. 


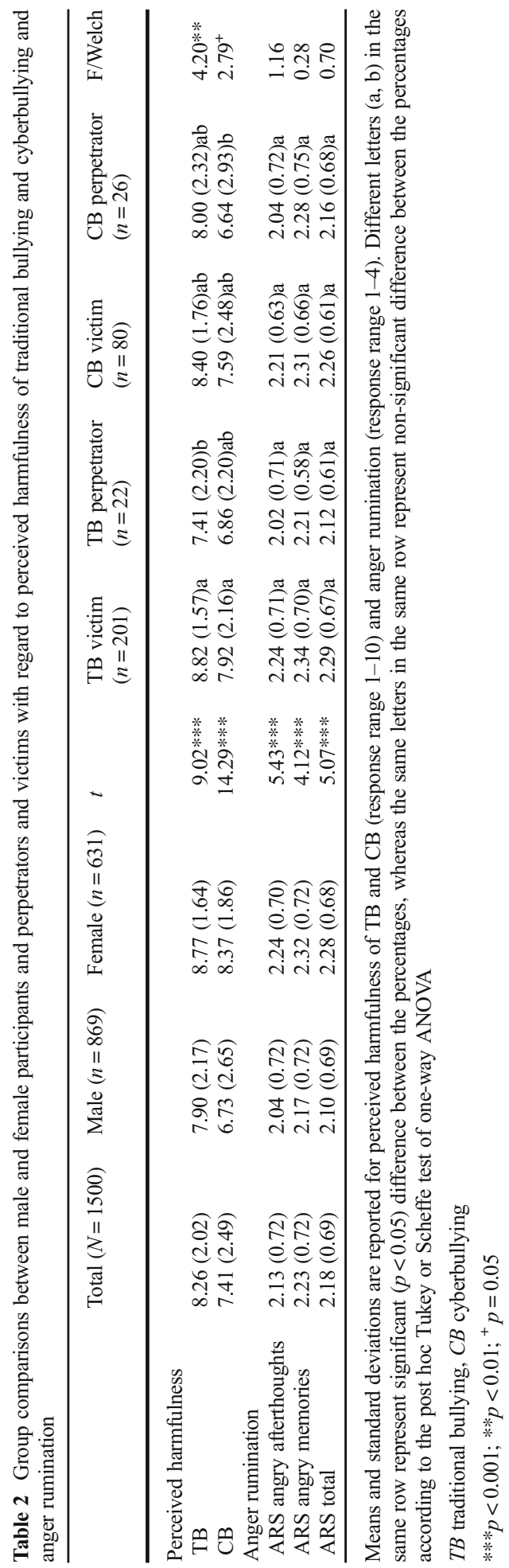




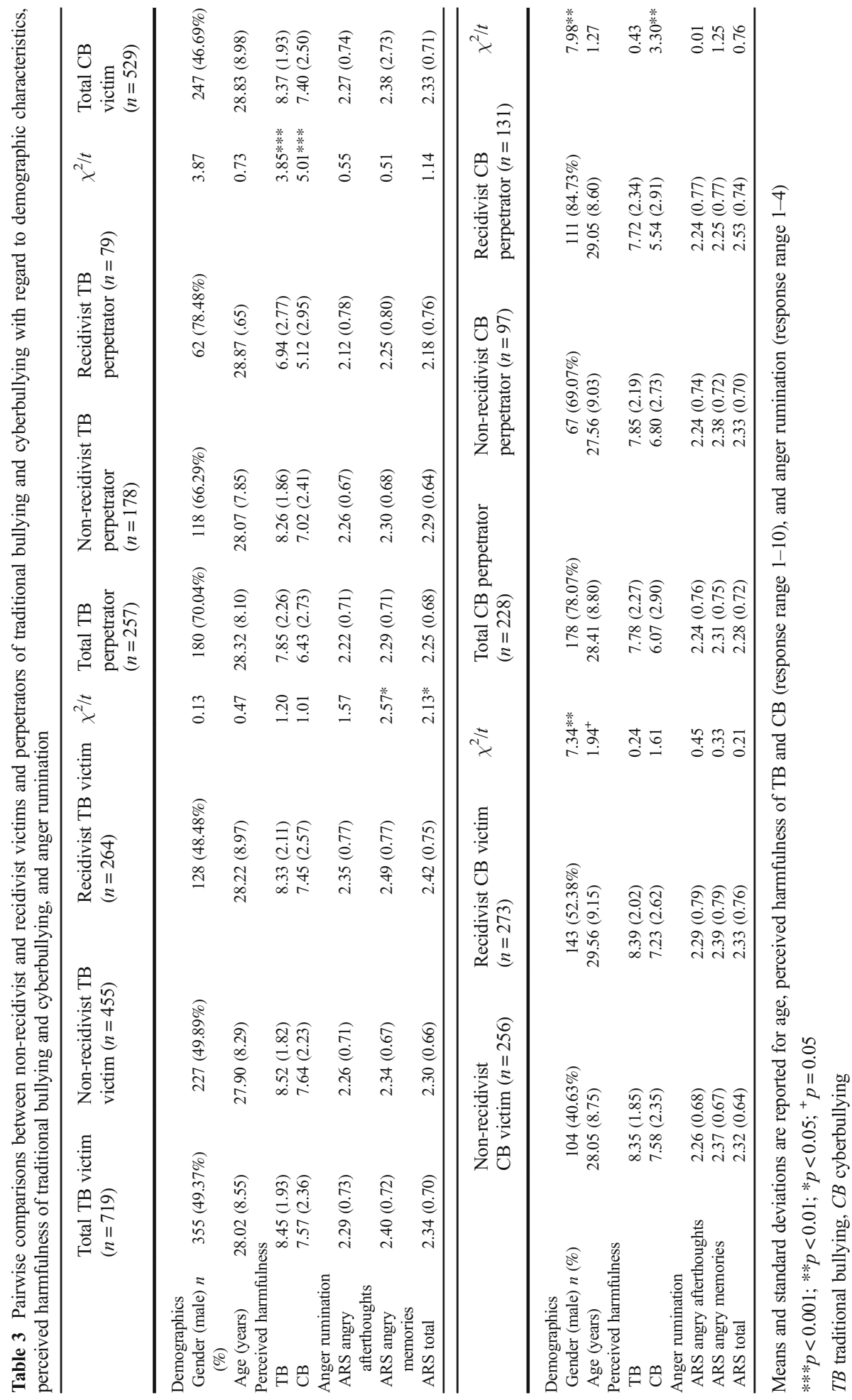




\section{The Moderation Models}

The role of anger rumination and repeated TB victimization and perpetration was investigated in the association between past $\mathrm{CB}$ victimization and recent $\mathrm{CB}$ perpetration separately for male and female participants. Seven moderation models were tested in which past $C B$ victimization was entered as an independent variable and recent $\mathrm{CB}$ perpetration as an outcome variable. Age was treated as a control variable and was entered as a covariate in the analyses.

H1 It was tested whether gender moderated the relationship between past $\mathrm{CB}$ victimization and recent $\mathrm{CB}$ perpetration. The interaction term was significant $(B=-1.09$ [ -1.85 to 0.32$]$; $p=0.006$ ), indicating that past $\mathrm{CB}$ victimization increased the risk of being a $\mathrm{CB}$ perpetrator in males (see Fig. 1). Therefore, the first hypothesis was supported.

H2 It was tested whether anger rumination moderated the relationship between past $\mathrm{CB}$ victimization and recent $\mathrm{CB}$ perpetration. When testing the moderator effect of anger rumination among males in this association, the interaction term was significant $(B=-0.75[-1.45$ to 0.06 ]; $p=0.03$ ), indicating that male $\mathrm{CB}$ victims who tended to ruminate about past anger experiences were more likely to become a $\mathrm{CB}$ perpetrator (see Fig. 2). In contrast, the moderating role of anger rumination in this association was not demonstrated in females because the interaction term emerged as non-significant $(B=-0.12[-1.01$ to 0.77$] ; p=0.79)$. Therefore, the second hypothesis was supported.

H3 The possible moderating role of repeated TB perpetration was tested in the association between past $\mathrm{CB}$ victimization and recent $\mathrm{CB}$ perpetration. However, the interaction term was neither significant among males $(B=0.08[-0.02$ to 0.18$] ; p=0.11)$ nor among females $(B=$ 0.10 [ -0.06 to 0.26$] ; p=0.21)$. Therefore, the third hypothesis was not supported.

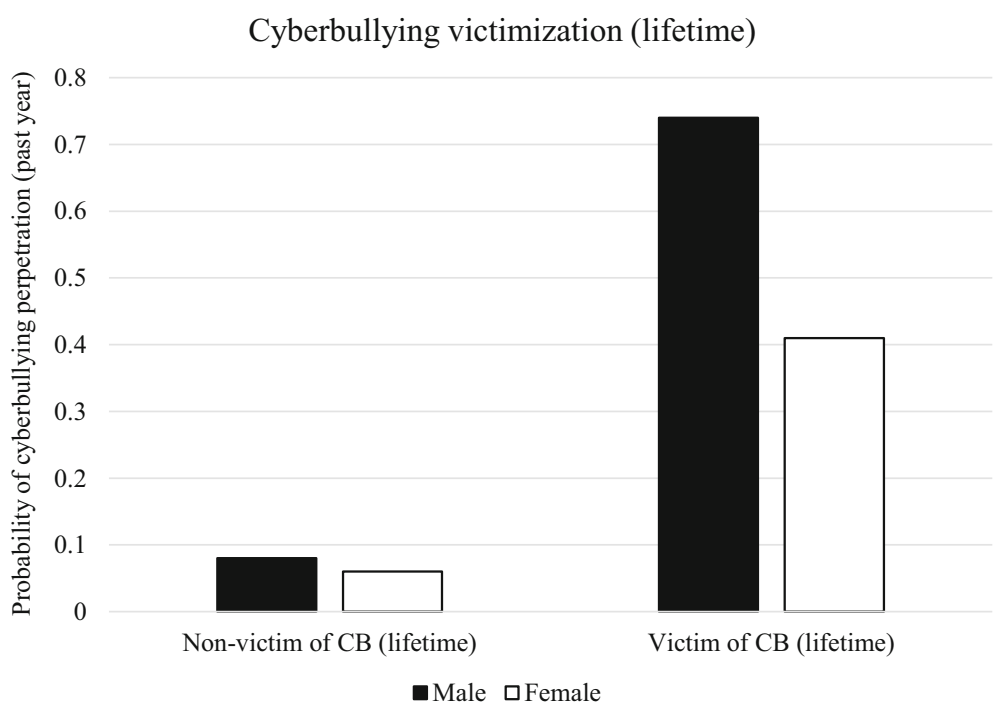

Fig. 1 The moderating effect of gender on the relationship between past cyberbullying (CB) victimization (lifetime) and recent cyberbullying perpetration (past year) 


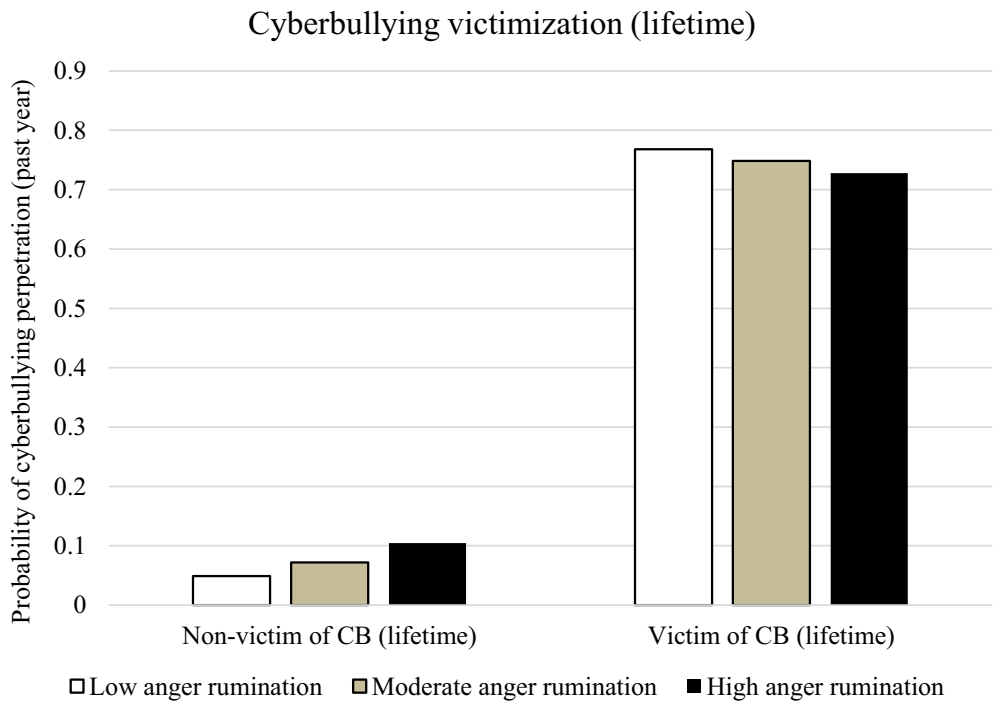

Fig. 2 The moderating effect of anger rumination on the relationship between past cyberbullying (CB) victimization (lifetime) and recent cyberbullying perpetration (past year) among male participants $(n=869)$

H4 The moderating role of repeated TB victimization was tested in the association between past $\mathrm{CB}$ victimization and recent $\mathrm{CB}$ perpetration. When testing the moderating role of repeated TB victimization among males, the interaction term was not significant $(B=-0.17$ $[-0.85$ to 0.51$] ; p=0.62)$. However, the interaction term emerged as significant among females in this association $(B=1.26[0.20$ to 2.31$] ; p=0.02)$. This result indicates that female $\mathrm{CB}$ victims with a recidivist $\mathrm{TB}$ victim history were more likely to become a $\mathrm{CB}$ perpetrator (see Fig. 3). Therefore, the fourth hypothesis was supported.

\section{Discussion}

Findings of prior investigations into the similarities and differences in traditional bullying (TB) and cyberbullying (CB) experiences have indicated relevant gender differences in the underlying psychological mechanisms (e.g., motives, perception, reaction) of victimization and perpetration (e.g., Fletcher et al. 2014; Hemphill and Heerde 2014; Waasdorp and Bradshaw 2015). Nevertheless, relatively little research attention has been paid to the possibly differential role of $\mathrm{TB}$ experiences and anger for males and females in the association of $\mathrm{CB}$ victimization and perpetration. Findings of the present study indicated that males were more likely than females to engage in $\mathrm{CB}$ behavior when they had previously been bullied online. Furthermore, anger ruminative tendencies elevated the risk of $\mathrm{CB}$ perpetration among males with a history of past $\mathrm{CB}$ victimization. Although repeated TB perpetration did not moderate the relationship between past $\mathrm{CB}$ victimization and recent $\mathrm{CB}$ perpetration, repeated $\mathrm{TB}$ victimization increased the risk of $\mathrm{CB}$ perpetration among female $\mathrm{CB}$ victims. These results provide further evidence for the differential psychological mechanisms underlying the motives of $\mathrm{CB}$ perpetration and the subsequent reactions to $\mathrm{CB}$ victimization in males and females. 


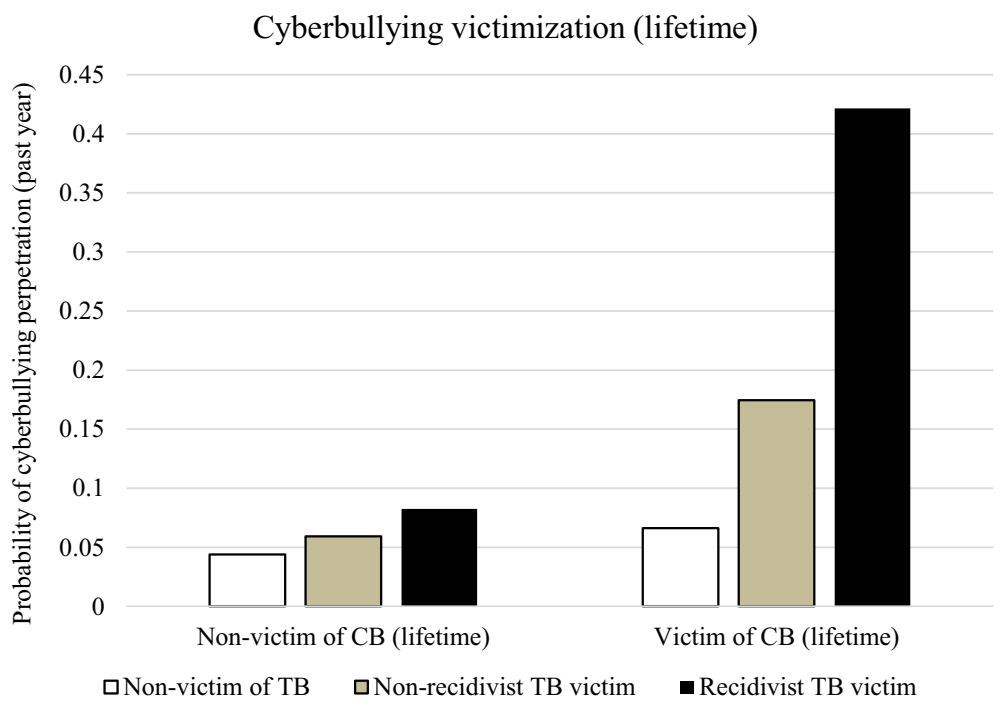

Fig. 3 The moderating effect of traditional bullying (TB) victimization on the relationship between past cyberbullying (CB) victimization (lifetime) and recent cyberbullying perpetration (past year) among female participants $(n=631)$

In the present study, almost half of adolescents and adults (47.9\%) reported of having been victimized in TB during their lifetime, whereas $35.3 \%$ had been bullied online. These prevalence rates are relatively higher than those reported by Modecki et al. (2014) in their meta-analysis. One possible explanation could be that individuals with personal involvement in bullying were more motivated to complete a survey focusing on bullying experiences than those who had never been involved in bullying.

In relation to gender, males were more likely to bully others offline and online. This result is in line with the findings of most studies in the literature (e.g., Chan and Wong 2016; Lee and Shin 2017). Furthermore, males harassed others more frequently than females and, in turn, were bullied more often online. These results also corroborate the findings reported in previous studies (e.g., Buelga et al. 2015; Kowalski et al. 2010), suggesting that males might have an elevated risk of being bullied by their victims who take revenge for being continuously harassed.

With regard to victimization, in the present study, more females reported having been victimized in TB and $\mathrm{CB}$. Most previous studies found that females are more likely to be a CB victim than males (e.g., DeHue et al. 2008; Ortega et al. 2009), while the reverse has been observed among TB victims (e.g., Huang and Chou 2010; Wang et al. 2009). Campbell et al. (2013) also reported of a higher proportion of female TB victims in their study and interpreted these higher rates as a possible consequence of using TB forms in the assessment that highly reflect indirect forms of aggression (e.g., exclusion) which are more prevalent among females (Hara 2002). The definition participants read of TB (Shaw et al. 2013) in the present study also contained a considerable proportion of indirect forms of aggression (e.g., spreading rumors, not allowing someone to join in an activity).

Similar to previous studies (e.g., Campbell et al. 2013; Hughes et al. 2009), females perceived $\mathrm{TB}$ and $\mathrm{CB}$ to be more harmful than males. Furthermore, in general, participants found TB to be more harmful than $\mathrm{CB}$, and as expected, victims perceived bullying more hurtful than perpetrators. These results were in line with those reported by Pettalia et al. (2013) and Campbell et al. (2013) who proposed that perpetrators are either not aware of the impact of their actions on 
victims or they ignore it. Also confirming this suggestion, recidivist perpetrators found $\mathrm{TB} / \mathrm{CB}$ less hurtful than non-recidivist perpetrators in the present study. This finding might indicate that some perpetrators continue bullying others because they perceive that the harm they cause is not so hurtful or they neglect the emotional consequences of their aggressive actions.

The first hypothesis received support because males were more likely to bully others online when they had experienced CB as a victim in their past. Previous studies have found an association between $\mathrm{CB}$ victimization and perpetration, and in accordance with the present results, males were more likely than females to become a CB perpetrator when they had also been victims (Wong et al. 2014; Wong et al. 2017). These findings may indicate that CB victimization has a differential role for males and females in becoming a $\mathrm{CB}$ perpetrator. More specifically, $\mathrm{CB}$ victimization elevates the risk of perpetration among males who might be more likely to retaliate for emotional hurt by exhibiting similar forms of aggression they experienced previously, while no such effect of past $C B$ victimization was observed among females. Therefore, possible determinants of $\mathrm{CB}$ perpetration should be examined separately for male and female victims, as previously suggested by Wong et al. (2017).

One of the possible determinants of TB and CB in the present study was anger rumination. A number of studies have found that males have a higher level of anger rumination than females (e.g., Maxwell et al. 2005; Peled and Moretti 2010), although other studies (e.g., Maxwell 2004; Sukhodolsky et al. 2001) reported no gender difference in anger rumination. In the present study, the moderating role of anger rumination in the association between past $\mathrm{CB}$ victimization and recent $\mathrm{CB}$ perpetration was demonstrated only among males, indicating that males who tend to ruminate about past experiences of anger are more likely to become a bully when experiencing bullying as a victim. Therefore, the second hypothesis was confirmed. Given that males have been found to exhibit more aggressive behaviors than females (see Bettencourt and Miller 1996 for a review) and are more likely to react to stressful events (e.g., conflicts) using a direct confrontation approach over avoidance (Taylor et al. 2000; Turton and Campbell 2005), it might be the case that those male victims who tend to ruminate about past angry episodes are more likely to fantasize about revenge (Sukhodolsky et al. 2001), and that such hostile thoughts might lead to a greater probability of actual retaliation.

With regard to TB experiences, repeated TB victimization, but not perpetration, moderated the relationship between past $\mathrm{CB}$ victimization and recent $\mathrm{CB}$ perpetration, and only among females. Therefore, the third hypothesis did not receive support. A similar pattern was observed in the study by Cappadocia et al. (2013) in which TB victimization, but not perpetration, predicted $\mathrm{CB}$ perpetration and victimization. This result might indicate that more experiences in the role of a TB perpetrator do not elevate the risk of engagement in online aggression as a new extension of aggressive behaviors. Instead, victimization is more strongly associated with intentions to offend.

Findings also suggested that repeated TB victimization elevated the risk of CB perpetration among females who were bullied online in the past. Therefore, the fourth hypothesis was supported. One possible explanation for this association could be that females are more likely to engage in CB than in TB for its anonymous and more indirect nature (Yang et al. 2013) and appeared to be more sensitive to the psychological distress caused by bullying compared to males. Therefore, repeated victimization in more forms of bullying (i.e., offline and online) might motivate female victims to take revenge for the harm caused in more indirect and less risky forms of retaliation (Cappadocia et al. 2013).

The present study has a number of limitations that should be acknowledged. First, the study's self-selected sample may not be representative of the entire population of Hungarian 
adolescent and adults; therefore, the generalization of the findings to both Hungarians more generally and non-Hungarians is compromised. Second, the cross-sectional nature of the study design constitutes a serious limitation as casual inferences for the associations reported cannot be made. Third, the retrospective self-report nature of some items in the assessment of past experiences of bullying might be biased (due to inaccurate memory recall or social desirability); therefore, longitudinal studies are necessary to reduce the effect of memory bias on the results. Fourth, the tested models were selected based upon theoretical considerations that reflected the conclusions of previous empirical investigations, but could be alternatively formulated (e.g., past CB perpetration might be considered as a predictor of recent CB victimization). Hence, alternative models should also be tested in future research.

Despite the limitations, findings of the present study may contribute to a better understanding of gender differences in motives, perception, and reaction to $\mathrm{CB}$ victimization that could possibly lead to hostile online behaviors. These results also highlight the importance of considering gender differences when investigating the effect of various psychological determinants concerning perpetration and victimization because substantial differences were observed in the determinants across gender. Furthermore, prevention and intervention efforts may benefit from using a more targeted approach to alleviate anger and negative emotions in male and female victims that experience different forms of bullying, in order to reduce the risk of future offending.

Funding Information This study was supported by the National Research, Development and Innovation Office (Grant number: K111938). Ágnes Zsila was supported by the ÚNKP-17-3 New National Excellence Program of the Ministry of Human Capacities.

\section{Compliance with Ethical Standards}

Conflict of Interest The authors declare that they have no conflict of interest.

Ethical Approval All procedures performed in this study involving human participants were in accordance with the ethical standards of University's Research Ethics Board and with the 1975 Helsinki Declaration.

Informed Consent Informed consent was obtained from all participants.

Open Access This article is distributed under the terms of the Creative Commons Attribution 4.0 International License (http://creativecommons.org/licenses/by/4.0/), which permits unrestricted use, distribution, and reproduction in any medium, provided you give appropriate credit to the original author(s) and the source, provide a link to the Creative Commons license, and indicate if changes were made.

\section{References}

Aluede, O., Adeleke, F., Omoike, D., \& Afen-Akpaida, J. (2008). A review of the extent, nature, characteristics and effects of bullying behaviour in schools. Journal of Instructional Psychology, 35(2), 151-159.

Balakrishnan, V. (2015). Cyberbullying among young adults in Malaysia: The roles of gender, age and Internet frequency. Computers in Human Behavior, 46, 149-157.

Barlett, C., \& Coyne, S. M. (2014). A meta-analysis of sex differences in cyber-bullying behavior: The moderating role of age. Aggressive Behavior, 40(5), $474-488$.

Beaton, D. E., Bombardier, C., Guillemin, F., \& Ferraz, M. B. (2000). Guidelines for the process of cross-cultural adaptation of self-report measures. Spine, 25(24), 3186-3191.

Bettencourt, B., \& Miller, N. (1996). Gender differences in aggression as a function of provocation: A metaanalysis. Psychological Bulletin, 119(3), 422-447. 
Buelga, S., Iranzo, B., Cava, M.-J., \& Torralba, E. (2015). Psychological profile of adolescent cyberbullying aggressors [Perfil psicosocial de adolescentes agresores de cyberbullying]. Revista de Psicología Social, 30(2), 382-406.

Bushman, B. J., Bonacci, A. M., Pedersen, W. C., Vasquez, E. A., \& Miller, N. (2005). Chewing on it can chew you up: Effects of rumination on triggered displaced aggression. Journal of Personality and Social Psychology, 88(6), 969-983.

Camodeca, M., Goossens, F. A., Terwogt, M. M., \& Schuengel, C. (2002). Bullying and victimization among schoolage children: stability and links to proactive and reactive aggression. Social Development, 11(3), 332-345.

Campbell, M. A., Slee, P. T., Spears, B., Butler, D., \& Kift, S. (2013). Do cyberbullies suffer too? Cyberbullies' perceptions of the harm they cause to others and to their own mental health. School Psychology International, 34(6), 613-629.

Cappadocia, M. C., Craig, W. M., \& Pepler, D. (2013). Cyberbullying: prevalence, stability, and risk factors during adolescence. Canadian Journal of School Psychology, 28(2), 171-192.

Chan, H. C., \& Wong, D. S. (2016). Traditional school bullying and cyberbullying perpetration: Examining the psychosocial characteristics of Hong Kong male and female adolescents. Youth \& Society, 1-27. Epub ahead of print. https://doi.org/10.1177/0044118X16658053.

Cohen, J. (1988). Statistical power analysis for the behavioral sciences. Hillsdale: Lawrence Earlbaum Associates.

DeHue, F., Bolman, C., \& Völlink, T. (2008). Cyberbullying: Youngsters' experiences and parental perception. Cyberpsychology \& Behavior, 11(2), 217-223.

Dooley, J., Pyzalski, J., \& Cross, D. (2009). Cyberbullying versus face-to-face bullying. A theoretical and conceptual review. Zeitschrift fur Psychologie/Journal of Psychology, 217, 182-188.

Erdur-Baker, Ö. (2010). Cyberbullying and its correlation to traditional bullying, gender and frequent and risky usage of internet-mediated communication tools. New Media \& Society, 12(1), 109-125.

Fenaughty, J., \& Harré, N. (2013). Factors associated with distressing electronic harassment and cyberbullying. Computers in Human Behavior, 29(3), 803-811.

Field, A. (2009). Discover statistics using SPSS. London: Sage.

Fletcher, A., Fitzgerald-Yau, N., Jones, R., Allen, E., Viner, R. M., \& Bonell, C. (2014). Brief report: Cyberbullying perpetration and its associations with socio-demographics, aggressive behaviour at school, and mental health outcomes. Journal of Adolescence, 37(8), 1393-1398.

Griffiths, M. D. (2014). Adolescent trolling in online environments: A brief overview. Education and Health, 32, $85-87$.

Hara, H. (2002). Justifications for bullying among Japanese schoolchildren. Asian Journal of Social Psychology, 5(3), 197-204.

Hayes, A. F. (2013). Introduction to mediation, moderation, and conditional process analysis: A regressionbased approach. New York: Guilford Press.

Hemphill, S. A., \& Heerde, J. A. (2014). Adolescent predictors of young adult cyberbullying perpetration and victimization among Australian youth. Journal of Adolescent Health, 55(4), 580-587.

Hemphill, S. A., Kotevski, A., Tollit, M., Smith, R., Herrenkohl, T. I., Toumbourou, J. W., \& Catalano, R. F. (2012). Longitudinal predictors of cyber and traditional bullying perpetration in Australian secondary school students. Journal of Adolescent Health, 51(1), 59-65.

Hinduja, S., \& Patchin, J. W. (2008). Cyberbullying: An exploratory analysis of factors related to offending and victimization. Deviant Behavior, 29(2), 129-156.

Huang, Y.-y., \& Chou, C. (2010). An analysis of multiple factors of cyberbullying among junior high school students in Taiwan. Computers in Human Behavior, 26(6), 1581-1590.

Hughes, P. P., Middleton, K. M., \& Marshall, D. D. (2009). Students' perceptions of bullying in Oklahoma public schools. Journal of School Violence, 8(3), 216-232.

Kowalski, R. M., \& Limber, S. P. (2007). Electronic bullying among middle school students. Journal of Adolescent Health, 41(6), S22-S30.

Kowalski, R. M., \& Limber, S. P. (2013). Psychological, physical, and academic correlates of cyberbullying and traditional bullying. Journal of Adolescent Health, 53(1), S13-S20.

Kowalski, R. M., Limber, S., \& Agatston, P. W. (2010). Cyber bullying: El acoso escolar en la era digital. Bilbao: Desclée de Brouwer.

Kowalski, R. M., Morgan, C. A., \& Limber, S. P. (2012). Traditional bullying as a potential warning sign of cyberbullying. School Psychology International, 33(5), 505-519.

Kowalski, R. M., Giumetti, G. W., Schroeder, A. N., \& Lattanner, M. R. (2014). Bullying in the digital age: A critical review and meta-analysis of cyberbullying research among youth. Psychological Bulletin, 140(4), 1073-1137.

LaGrange, T. C., \& Silverman, R. A. (1999). Low self-control and opportunity: Testing the general theory of crime as an explanation for gender differences in delinquency. Criminology, 37(1), 41-72. 
Lee, C., \& Shin, N. (2017). Prevalence of cyberbullying and predictors of cyberbullying perpetration among Korean adolescents. Computers in Human Behavior, 68, 352-358.

Li, Q. (2007). New bottle but old wine: A research of cyberbullying in schools. Computers in Human Behavior, 23(4), 1777-1791.

MacDonald, C. D., \& Roberts-Pittman, B. (2010). Cyberbullying among college students: Prevalence and demographic differences. Procedia-Social and Behavioral Sciences, 9, 2003-2009.

Maxwell, J. (2004). Anger rumination: An antecedent of athlete aggression? Psychology of Sport and Exercise, 5(3), 279-289.

Maxwell, J. P., Sukhodolsky, D. G., Chow, C. C., \& Wong, C. F. (2005). Anger rumination in Hong Kong and Great Britain: Validation of the scale and a cross-cultural comparison. Personality and Individual Differences, 39(6), 1147-1157.

Modecki, K. L., Minchin, J., Harbaugh, A. G., Guerra, N. G., \& Runions, K. C. (2014). Bullying prevalence across contexts: A meta-analysis measuring cyber and traditional bullying. Journal of Adolescent Health, $55(5), 602-611$.

Olweus, D. (1999). Sweden. The nature of school bullying: A cross-national perspective. London: Routledge.

Olweus, D. (2012). Cyberbullying: An overrated phenomenon? European Journal of Developmental Psychology, $9(5), 520-538$.

Ortega, R., Elipe, P., Mora-Merchan, J. A., Calmaestra, J., \& Vega, E. (2009). The emotional impact on victims of traditional bullying and cyberbullying: A study of Spanish adolescents. Zeitschrift fur Psychologie/ Journal of Psychology, 217, 197-204.

Ortega, R., Elipe, P., Mora-Merchán, J. A., Genta, M. L., Brighi, A., Guarini, A., et al. (2012). The emotional impact of bullying and cyberbullying on victims: A European cross-national study. Aggressive Behavior, $38(5), 342-356$.

Peled, M., \& Moretti, M. M. (2010). Ruminating on rumination: Are rumination on anger and sadness differentially related to aggression and depressed mood? Journal of Psychopathology and Behavioral Assessment, 32(1), 108-117.

Pettalia, J. L., Levin, E., \& Dickinson, J. (2013). Cyberbullying: Eliciting harm without consequence. Computers in Human Behavior, 29(6), 2758-2765.

Shaw, T., Dooley, J. J., Cross, D., Zubrick, S. R., \& Waters, S. (2013). The Forms of Bullying Scale (FBS): Validity and reliability estimates for a measure of bullying victimization and perpetration in adolescence. Psychological Assessment, 25(4), 1045-1057.

Simourd, L., \& Andrews, D. A. (1994). Correlates of delinquency: A look at gender differences. Forum on Corrections Research, 6(1), 26-31.

Smith, P. K., Mahdavi, J., Carvalho, M., Fisher, S., Russell, S., \& Tippett, N. (2008). Cyberbullying: Its nature and impact in secondary school pupils. Journal of Child Psychology and Psychiatry, 49(4), 376-385.

Sukhodolsky, D. G., Golub, A., \& Cromwell, E. N. (2001). Development and validation of the anger rumination scale. Personality and Individual Differences, 31(5), 689-700.

Taylor, S. E., Klein, L. C., Lewis, B. P., Gruenewald, T. L., Gurung, R. A., \& Updegraff, J. A. (2000). Biobehavioral responses to stress in females: Tend-and-befriend, not fight-or-flight. Psychological Review, 107(3), 411-429.

Thacker, S., \& Griffiths, M. D. (2012). An exploratory study of trolling in online video gaming. International Journal of Cyber Behavior, Psychology and Learning, 2(4), 17-33.

Tokunaga, R. S. (2010). Following you home from school: A critical review and synthesis of research on cyberbullying victimization. Computers in Human Behavior, 26(3), 277-287.

Turton, S., \& Campbell, C. (2005). Tend and befriend versus fight or flight: Gender differences in behavioral response to stress among university students. Journal of Applied Biobehavioral Research, 10(4), $209-232$.

Waasdorp, T. E., \& Bradshaw, C. P. (2015). The overlap between cyberbullying and traditional bullying. Journal of Adolescent Health, 56(5), 483-488.

Wang, J., Iannotti, R. J., \& Nansel, T. R. (2009). School bullying among adolescents in the United States: Physical, verbal, relational, and cyber. Journal of Adolescent Health, 45(4), 368-375.

Wong, D. S., Chan, H. C. O., \& Cheng, C. H. (2014). Cyberbullying perpetration and victimization among adolescents in Hong Kong. Children and Youth Services Review, 36, 133-140.

Wong, R. Y., Cheung, C. M., \& Xiao, B. (2017). Does gender matter in cyberbullying perpetration? An empirical investigation. Computers in Human Behavior, 79, 247-257.

Yang, S.-J., Stewart, R., Kim, J.-M., Kim, S.-W., Shin, I.-S., Dewey, M. E., et al. (2013). Differences in predictors of traditional and cyber-bullying: A 2-year longitudinal study in Korean school children. European Child \& Adolescent Psychiatry, 22(5), 309-318. 